\title{
EL DESAFÍO DE UNA DEFINICIÓN SECULAR DE LA PERSONA PARA LA ÉTICA*
}

Bernard Schumacher*

La sociedad occidental se ve confrontada en el amanecer del tercer milenio a toda una serie de desafíos bioéticos que apelan a determinada concepción de la persona humana, desafíos tales como el aborto, la utilización con fines experimentales de embriones humanos, el infanticidio de niños muy gravemente impedidos mental y/o físicamente, e incluso la eutanasia. Las respuestas filosóficas a estos desafíos referidas a la tradición judeo-cristiana están, cada vez más, tomadas por asalto por una concepción ética post-metafísica que se inserta en el actual proceso de secularización de la sociedad occidental. Un número creciente de filósofos está pidiendo una transformación copernicana de los principios éticos de tradición judeo-cristiana que han impregnado profundamente a la sociedad occidental, y plantea una pregunta antropológica central: ¿todos los seres humanos son personas? Anne Fagot, dedicada a bioética en el Collège de France, nos lleva a poner atención a la apuesta fundamental subyacente: "hay conflicto entre el principio del respeto debido al ser humano y la instrumentalización de dicho ser humano en los estadios embrionarios y fetales, a menos que un embrión humano no sea una persona humana". ${ }^{1}$ Cons-

* Universidad de Friburg, Departamento de Filosofía. Traduccion del francés por Silvia Pasternac.

${ }^{1}$ A. Fagot y G. Delaisi de Parseval, "Les droits de l'embryon (foetus) humain, et la personne humaine potentielle" ["Los derechos del embrión (feto) humano, y la persona humana potencial"], Revue de Métaphysique et de Morale, 1987 (92), n 3, p. 362. 
ciente de esta apuesta, cierto número de filósofos ha decidido ya no otorgarle la condición de persona a todo ser humano, con la esperanza de resolver con esto problemas bioéticos espinosos. Me gustaría concentrar mi atención en uno de los especialistas más influyentes del mundo anglosajón, Tristam Engelhardt. Después de presentar su teoría de una ética secular, desarrollaré su comprensión de la persona, que él diferencia del ser humano no moral, así como la contra-argumentación referente a la noción de persona potencial. Luego discutiré sobre la legitimidad del concepto de persona social otorgado a los seres humanos nacidos que no son personas morales, para concluir con una proposición de antropología unitaria.

\section{La ética secular}

"Después de haber dominado nuestros pensamientos y nuestras decisiones concernientes a la vida y la muerte durante casi dos mil años, la ética occidental tradicional se ha derrumbado."2 Peter Singer se refiere aquí a la ética de tradición judeo-cristiana, que él describe como fundamentalmente incoherente, como una 'farsa' y una 'tragedia'. Unas cuantas páginas más adelante, precisa su pensamiento:

La ética tradicional es defendida todavía por obispos y bioéticos conservadores que hablan con tono reverente del valor de toda vida humana, independientemente de su naturaleza o de su calidad. Sin embargo, como el traje nuevo del emperador, estas frases solemnes parecen ser verdaderas y sustanciales solamente en la medida en que somos intimidados para una aceptación desprovista de críticas de la afirmación de que toda vida humana tiene una dignidad o un valor particular. Cuando es desafiada, la ética tradicional se derrumba. [...] tenemos una oportunidad histórica de elaborar algo mejor, una ética que no necesite sostenerse en ficciones límpidas en las que nadie puede

${ }^{2}$ Ver Peter Singer, Rethinking Life and Death. The Collapse of our Traditional Ethics, 1994, Nueva York, St. Martin's Press. 
creer verdaderamente, una ética que es más compasiva y receptiva a lo que las personas deciden para sí mismas. ${ }^{3}$

Esta exhortación a una transformación radical de los principios que rigen la ética y, más particularmente, la bioética es retomada por un número creciente de filósofos occidentales. En su última obra, titulada Féeries anatomiques. Généalogie du corps faustien, Michel Onfray, por ejemplo, lanza un llamado apremiante -desprovisto, sin embargo, de argumentos filosóficos- en favor de una biopolítica libertaria que invite a toda persona a decidir por voluntad propia los hechos establecidos por la bioética. Tomando distancia tanto de una heurística del miedo con respecto a la restauración del superhombre nazi y de la eugenesia, como del Principio de responsabilidad desarrollado por Hans Jonas, que impregnan a los comités de éticos occidentales, Onfray propone una heurística de la audacia que es, en su opinión, un simple postulado que no necesita demostración ni justificación, ${ }^{4}$ y que implica el rechazo de la tradición ética judeo-cristiana. ${ }^{5}$ Onfray se constituye en el promotor de una bioética secular donde todo sería facultativo, donde cada uno sería libre de comprometerse en el terreno de su propia decisión. "Aumentar las posibilidades, precisa, no fuerza a nadie a efectuar una elección que lastime su moral."6 Onfray resume así, me parece, la clave que resolvería los espinosos problemas relativos a la vida

${ }^{3}$ Ibid., 4: "The traditional ethic is still defended by bishops and conservative bioethicists who speak in reverent tones about the intrinsic value of all human life, irrespective of its nature or quality. But, like the new clothes worn by the emperor, these solemn phrases seem true ans substantial only while we are intimidated into uncritically accepting that all human life has some special dignity or worth. Once challenged, the traditional ethic crumples. [...] we have an historic chance to shape something better, an ethic that does not need to be propped up by transparent fictions, no-one can really believe, an ethics that is more compassionate and more responsive to what people decide for themselves." Ver p. 220-1.

${ }^{4}$ Ver Michel Onfray, Féeries anatomiques. Généalogie du corps faustien [Cuentos de hadas anatómicos. Genealogía del cuerpo faustiano], 2003, París, Grasset, p. 80.

${ }^{5}$ Ver ibid., p. 88. Onfray precisa que "cada línea de mis libros proviene de una voluntad feroz de descristianizar a la civilización en la cual pasamos furtivamente entre dos vacíos", p. 89.

${ }^{6}$ Ibid., p. 72. 
y la muerte en un contexto secular y neutro con respecto a las asunciones éticas judeo-cristianas, a saber, en un entorno post-cristiano, como por otro lado lo describe Engelhardt. ${ }^{7}$ Este último había propuesto una clave de lectura similar casi veinte años antes. Constata, de acuerdo con John Rawls, que la sociedad occidental es esencialmente pluricultural. Los puntos de vista de la moralidad llamada sustancial pertenecen a comunidades particulares de creencias cuyos miembros comparten las mismas metas y valores. Todos los intentos del proyecto filosófico moderno de establecer con la ayuda de argumentos racionales una teoría ética verdadera y universal que constituya el fundamento de la paz perpetua se han revelado imposibles. En el amanecer del tercer milenio, la bioética sigue siendo profundamente plural. Ninguna visión particular de bioética podría asumir la totalidad de las aproximaciones presentes en el mundo occidental. ${ }^{8}$ La capitulación de la razón filosófica con vistas a establecer una ética universal podría, sin embargo, superarse si nos refiriéramos a un Dios trascendente, que implicara la creencia en una Revelación. Engelhardt, quien, por su parte, es un creyente ortodoxo, rechaza esta solución, pues hace referencia a una creencia que no comparten todas las personas morales de la comunidad humana.

Con el fin de resolver este dilema y garantizar una base racional a la esperanza de una vida comunitaria en paz, Engelhardt propone la instauración de una ética secular. Para hacerlo, distingue dos tipos de comunidades: la primera, compuesta por 'amigos morales', comparte una ética común basada en valores y axiomas idénticos, y está en condiciones de resolver cierto número de controversias éticas; la segunda, compuesta de 'extranjeros morales', no comparte suficientes premisas como para ser capaz de resolver debates éticos. ${ }^{9}$ Se trataría entonces de emplazar una plataforma neutra con un discurso ético muy amplio y pragmático, que permitiría guiar a los 'extranjeros morales' a la reali-

7 Ver Tristam Engelhardt Jr., "Infanticide in a Post-Christian Age", MacMillan, Engelhardt y Spicker (eds.), Euthanasia and the Newborn, 1987, Dordrecht, Reidel, p. 81. The Foundations of Bioethics, 1996, 2a., Oxford, Oxford University Press, p. 6.

${ }^{8}$ Ver Engelhardt, The Foundations..., op. cit., p. 10-1.

${ }^{9}$ Ibid., p. 7. 
zación de una política de la atención alrededor del principio de la tolerancia con respecto a las éticas particulares, es decir, que respetaría profundamente las convicciones éticas de las otras personas morales a pesar de sus diferencias profundas. Cada individuo autónomo y cada comunidad tienen derecho a vivir de acuerdo con sus propios principios éticos. Ninguna comunidad particular de personas morales podría imponer su propio punto de vista a expensas de las demás comunidades. Si el Estado tuviera que prescribir una teoría particular del bien, lo haría, según Engelhardt, recurriendo a la constricción, actitud que conduciría al fin de la democracia, a una situación de la época anterior a la Ilustración, con poderes inquisitoriales en la vida privada de los ciudadanos. ${ }^{10}$ El principio de tolerancia de una ética secular, que es necesariamente permisiva, salvaguarda, sin embargo, derechos y deberes particulares relativos a cada comunidad singular y permite así, en opinión de Engelhardt, instaurar una vida comunitaria en paz. Nuestro filósofo precisa que

La paz perpetua en ausencia de represión llegará probablemente, si es que llega, cuando nos pongamos de acuerdo para apoyar las elecciones que realizan las personas consigo mismas, con sus recursos privados, con otras personas que estén de acuerdo con dicha elección, y con sus comunidades, por más divergentes que éstas sean, e incluso si las elecciones son profundamente falsas. ${ }^{11}$

\section{La condición de la persona en una ética secular}

La comunidad de los 'extranjeros morales' que elabora en conjunto una ética secular está compuesta por personas, es decir, agentes morales autónomos y responsables, capaces de escoger libremente, de cerrar acuerdos entre ellos y de decidir sobre las reglas morales. Estas aptitudes

${ }^{10}$ Ver ibid. "Introduction", Dondeson, Engelhardt y Spicker (eds.), Abortion and the Status of the Foetus, 1983, Dordrecht, Reidel, p. XIX.

${ }^{11}$ Engelhardt, The Foundations..., op. cit., p. 15. 
presuponen la racionalidad y la autoconciencia. La persona es definida, a partir de esto, como un individuo racional, auto-conciente y libre que posee un sentido moral. ${ }^{12}$ Siguiendo a Emmanuel Kant, Engelhardt entiende a la persona como esencialmente una persona moral: un ser capaz por su racionalidad y su autoconciencia de apreciar que sus acciones pueden ser percibidas desde el ángulo de la reprobación o del elogio, o de estar en condiciones de realizar elecciones racionales y ser responsable en cuanto a su actuar. Sólo los individuos que posean en acto las propiedades mencionadas más arriba son personas propiamente dichas, que se respetan mutuamente y que participan en el emplazamiento de una comunidad moral secular de paz. Las personas son la condición y el fin de semejante orden ético secular. Son los actores privilegiados de la elaboración de las reglas éticas comunes, de un discurso moral, así como los sujetos de las reprobaciones y de las recompensas. Además de tener, sin duda, un valor, la persona posee ante todo una dignidad inviolable. Debe ser tratada, como lo subraya el segundo imperativo categórico de Emmanuel Kant, como un fin en sí, y no simplemente como un medio.

Una vez planteada esta definición de la persona moral, Engelhardt introduce, siguiendo a John Locke, una diferencia ontológica entre ser humano y persona. El ser humano no necesariamente es ya una persona por su sola pertenencia a la especie humana. Las características biológicas humanas son secundarias en cuanto al otorgamiento de la condición de persona. Engelhardt precisa que

Lo que distingue a las personas es su capacidad [en el sentido de ser en acto] de ser auto-concientes, racionales y preocupadas por el mérito de la reprobación o del elogio. [...] no todos los seres humanos son personas. No todos los seres humanos son auto-concientes, racionales y capaces de concebir la posibilidad de la reprobación y del elogio. Los fetos, los recién nacidos, los impedidos mentales muy profundos y los comatosos sin esperanza [y podríamos agregar los seniles] ofrecen

${ }^{12}$ Ibid., p. 136 s. "Medicine and the Concept of Person", M. F. Goodman (ed.), What is a person?, 1988, Clifton (N. J.), Humana Press, p. 171. 
ejemplos de no-personas humanas. Son miembros de la especie humana pero no tienen en y por sí mismas un lugar en la comunidad moral laica. $^{13}$

Con el trasfondo de esta distinción, prácticamente no tiene sentido hablar en términos de una moral secular de respeto de la autonomía de los seres humanos que no son personas. Estos individuos no pertenecen al corazón de la ética secular, y tienen muy poco valor moral. ${ }^{14}$ Engelhardt mantiene sobre esta base que "no todos los seres humanos son iguales". 15

El tratamiento moral de un ser humano depende así en primer lugar de su condición de persona que tiene un fin en sí misma. Sólo la persona posee derechos y deberes, así como una dignidad. ${ }^{16}$ Contrariamente a la persona autónoma que decide sola sobre su porvenir y sus propios intereses -es decir, que es auto-legisladora-el ser humano no-persona -tal como el embrión, el recién nacido, el impedido mental extremadamente grave y el senil-depende, según Engelhardt, enteramente de las personas morales que determinan lo que va en su mayor interés. En el caso de un conflicto de intereses, los de una persona privan necesariamente sobre los de una no-persona según criterios utilitaristas y consecuencialistas. ${ }^{17}$ La persona determina el valor del ser humano no-persona según el grado de sensación y de conciencia de metas. Si éste no ha desarrollado una vida consciente, la persona sólo le otorgará poco valor. El valor aumenta según el grado de vida interior en un sentido pre-reflexivo. La persona moral comparará sin embargo este

${ }^{13}$ Engelhardt, The Foundations..., op. cit., p. 138-9. Más adelante, Engelhardt incluye a los seniles entre las no-personas (p. 239).

14 "That mere human biological life is of little moral value in and of itself", ibid., p. 243.

${ }^{15}$ Ibid., p. 135.

16 "Insofar as we identify persons with moral agents, we exclude from the range of the concept of person those entities which are not self-conscious. Which is to say, only those beings are unqualified bearers of rights and duties who can both claim to be acknowledged as having a dignity beyond a value (i.e., as being ends in themselves), and can be responsible for their actions. [...] It is only respect for persons in this strict sense that cannot be violated without contradicting the idea of a moral order in the sense of living with others on the basis of mutual respect", Engelhardt, "Medicine and the concept of Person", op. cit., p. 172-3.

${ }^{17}$ Ver Engelhardt, The Foundations..., op. cit., p. 141. 
valor con otros valores competitivos de orden personal, tales como proyectos y deseos personales. ${ }^{18}$ Tomemos, por ejemplo, el caso de una pareja que ha deseado ardientemente durante años tener un hijo. La mujer embarazada otorgará al embrión un alto valor. En cambio, para la joven universitaria embarazada, el embrión podría significar la interrupción de varios proyectos que son esenciales en su opinión, tales como estudios y una carrera. El embrión tendría así un valor menor con relación a los proyectos de la joven mujer. Engelhardt agrega igualmente el hecho de que muchas personas de la sociedad occidental que deben participar en los costos de la educación de un niño trisómico le otorgan a este último un valor menor.

La dependencia del ser humano no-persona de las personas morales reposa sobre un a priori fundamental del pensamiento de Engelhardt, que él presenta al referirse al feto. Éste es comprendido como un objeto propiedad de las personas morales o de una sociedad anónima, que lo han producido: es "una forma particular de una propiedad muy cara". 19 Semejante afirmación reposa sobre una tesis no demostrada, y planteada a priori, de que el feto es una "extensión y el fruto de mi propio cuerpo". "Ellos [las personas morales] lo produjeron, lo hicieron, es de 54 ellos", 21 hasta

que ellos [los seres humanos no-personas] tomen posesión de sí mismos como entidades concientes, hasta que se les dé un lugar particular en una comunidad, hasta que una persona transfiera sus derechos sobre ellos a otra persona, o hasta que se conviertan en personas. ${ }^{22}$

${ }^{18}$ Ver ibid., p. 143: es necesario precisar que Engelhardt no habla aquí directamente de ser humano, sino de animal.

${ }^{19}$ Ibid., p. 255: "A special form of very dear property". "Those who made or procreated the zygote, embryo, or foetus have first claim on making the definitive determination of its value. Privately produced embryos and foetuses are private property. They would be societally owned only if societal groups or cooperatives produced them", p. 255. Ver p. 271.

20 "Extension(s) of and the fruit of one's own body", ibid., p. 256.

21 "They produced it, they made it, it is theirs", ibid., p. 255. Ver p. 271.

${ }^{22}$ Idem, p. 256. 
Al ser comprendido como un objeto, el ser humano feto no debería, en sí, ser tratado como un fin en sí, sino que podría ser utilizado simplemente como una cosa.

La consecuencia de esta posición es clara: es legítimo utilizar el feto con fines experimentales. Esta instrumentalización del feto, precisa Engelhardt, es igualmente válida para el cigoto y el embrión humanos, así como para el esperma y el óvulo humanos. La referencia al esperma y al óvulo verdaderamente es muy sorprendente, pues implica que su autor no distingue la diferencia esencial entre la especie de un ser humano y la especie de un esperma y de un óvulo humanos, que no son en cuanto tales seres humanos.

El esperma humano, el óvulo humano, los cultivos celulares humanos, los cigotos humanos, los embriones y el feto pueden tener un valor, pero están desprovistos de la dignidad de persona. Así, están, todos por igual, abiertos a una experimentación justificable socialmente de una manera en que las personas en su sentido estricto o social no deberían nunca estarlo. Esto significa que pueden ser utilizados simplemente como medios. $^{23}$

Engelhardt concluye lógicamente que no podría entonces haber, en el marco de una ética secular cuya piedra angular reposa sobre la distinción entre ser humano y persona, argumentos contra la experimentación no terapéutica de los cigotos, de los embriones, de los fetos, con vistas a un bien para la comunidad de las personas, a condición, sin embargo, de que los progenitores, o incluso una sociedad anónima, que son sus propietarios, den su acuerdo. ${ }^{24}$ A primera vista, esta posición parece estar sujeta, sin embargo, a una posible contra-argumen-

${ }^{23}$ Ver Engelhardt, "Medicine and the Concept of Person", op. cit., p. 179: En el contexto de su discusión sobre la definición de la muerte y el momento del deceso, Engelhardt precisa en el mismo artículo que "if such a body is an instance of human biological but not human personal life, then it is open to use merely as a subject of experimentation without the contraints of a second status as a person", p. 170. Engelhardt, The Foundations..., op. cit., p. 256, 271. Ver, con respecto a la definición de la muerte, B. Schumacher, Der Tod in der Philosophie der Gegenwart, 2004, Darmstadt, Wissenschaftliche Gesellschaft, primera parte.

${ }^{24}$ Ver Engelhardt, The Foundations..., op. cit., p. 272. 
tación. Si se acepta a priori lo fundamentado de la distinción entre ser humano y persona, se podría sostener, con el Comité consultivo nacional de Ética en Francia, ${ }^{25}$ que el embrión sería una persona potencial, es decir, que tendría fundamentalmente una potencialidad natural para convertirse en una persona, si las condiciones interiores y exteriores se cumplen. A partir de esto, sería sujeto con los mismos derechos que la persona moral.

\section{La persona potencial sujeta a una dignidad personal}

El argumento referente a la persona potencial sostiene que la atribución de derechos a X no depende de la posesión en acto por X de determinadas propiedades - cosa que le otorgaría el privilegio de pertenecer a la clase de persona-, sino de su potencialidad de poder un día disponer de ellas en acto, en el marco de su desarrollo natural. Los derechos relativos a la persona en acto serían así otorgadas por extensión al ser humano potencialmente personal. Entonces, la supresión de una persona potencial equivaldría a privarla de convertirse en una persona y a cometer un acto que iría en contra de un derecho fundamental de la persona: el de seguir viviendo. A pesar de que reconoce, ciertamente, que el feto es un ser humano con un potencial, Engelhardt se niega, sin embargo, a otorgarle derechos relativos a la persona. Éstos están únicamente reservados a la persona en acto, a saber, al individuo que ejerce actualmente las propiedades mencionadas más arriba. La poten-

${ }^{25}$ Ver "Avis relatif aux recherches sur les embryons humains in vitro et à leur utilisation à des fins médicales et scientifiques" ["Dictamen relativo a las investigaciones sobre los embriones humanos in vitro y a su utilización con fines médicos y científicos"]. Informe, 15 de diciembre de 1986 en CCNE, Avis de recherches sur l'embryon [Dictamen sobre investigaciones sobre el embrión, Arles, Acte Sud/INSERM, 1987, p. 78-178 o CCNE, Étique et recherche biomédicale, rapport 1986 [Ética e investigación biomédica, informe 1986], la documentation française, 1987, París, p. 27-94. M. A. Warren, "On the Moral and Legal Status of Abortion", The Monist, 1973 (57), p. 43-61, p. 59, y "Abortion", H. Kuhse y P. Singer (eds.), A Companion to Bioethics, 1988, Blackwell, Oxford, p. 127-134, p. 131. 
cialidad de poseerlas un día no implica en sí la exigencia lógica de ser sujeto a los derechos relativos a la persona en acto.

Pero si X es un Y potencial, de ello se deduce que X no es un Y. Si los fetos son personas potenciales, se deduce claramente de ello que los fetos no son personas. Por consiguiente, $\mathrm{X}$ no tiene los derechos actuales de $\mathrm{Y}$, sino que tiene sólo potencialmente los derechos de Y. Si los fetos no son más que personas potenciales, no tienen los derechos de personas. ${ }^{26}$

Engelhardt sostiene, con razón, que o bien $\mathrm{X}$ es ya una persona, $\mathrm{o}$ no lo es. No podría haber un punto medio, es decir que X no puede devenir en una persona, si no es ya una persona. En efecto, un ser no puede devenir a partir de algo que no era. Si ya lo era, no podría haber devenir en cuanto al ser. Sin embargo, nuestro autor confunde la doble significación del término 'persona potencial'. Éste puede entenderse -el primer sentido- como un ser humano que no es una persona, pero que es susceptible de devenir persona -nivel en el que se sitúa la contraargumentación de Engelhardt-, o -el segundo sentido- como un ser humano que ya es en acto una persona, pero que está tendiente a la actualización de sus potencialidades, que está destinado a desarrollar su potencial con vistas a su plena auto-realización. Ciertamente, podemos afirmar con Engelhardt que no hay, hablando con propiedad, persona potencial como tal, sino más bien una persona con un potencial. Sin embargo, al no concebir la segunda significación del término 'persona potencial', la posición de Engelhardt reposa sobre el a priori de lo que se podría describir en términos de desempeño y de éxito con respecto a la posesión en acto, en el ejercicio de propiedades llamadas personales. El ser humano que estuviera desprovisto de su ejercicio por una razón cualquiera no podría ser reconocido en el sentido estricto, en opinión de nuestro filósofo, como perteneciente a la clase de persona,

${ }^{26}$ Engelhardt, The Foundations..., op. cit., p. 142. "The potentiality of X's to become Y's may cause us to value X's very highly because Y's are valued very highly, but until X's are Y's they do not have the value of Y's." Engelhardt, "Medicine and the Concept of Person", op. cit., p. 174. 
y así gozar de los derechos relativos a ese grupo. Engelhardt omite distinguir entre el acto de ser una persona, por un lado y, por otro, el ejercicio y el comportamiento de esta persona, que se transparenta a través de su actuar, el ejercicio de una serie de propiedades llamadas personales. Semejante distinción permitiría concebir que un individuo humano pudiera ser en acto, a saber, una persona, sin ejercer necesariamente las propiedades que definen a la persona. Su ejercicio manifestado a otras personas no constituye el criterio que determinaría la pertenencia a la categoría de persona. Partiendo de esto, el ser humano no deviene en una persona por ejercer una serie de propiedades, tales como la autoconciencia, la racionalidad, la moralidad o la libertad, sino que es por su naturaleza, porque es fundamentalmente un ser humano-persona que tiene la potencialidad de ejercer dichas propiedades.

\section{La noción de persona social}

Como el feto y el embrión normal no han sido reconocidos como persona potencial -lo cual les hubiera permitido acceder a los derechos y a la dignidad personales-, son objetos que las personas morales pueden utilizar con fines experimentales, o matar, en la medida en que sus actos contribuyan a un acrecentamiento de la felicidad para la comunidad de los agentes morales. Sin embargo, ¿qué ocurre con la condición de los demás seres humanos no-personas, como el recién nacido y el adulto gravemente impedido mental o físicamente, como el senil? ¿Acaso no habría que mantener lógicamente que ellos también pueden, a su vez, estar sujetos a utilizaciones experimentales, al infanticidio y a la eutanasia? Engelhardt reconoce que estas acciones son contrarias al sentido común. Para salir del callejón sin salida en el que se encuentra, nuestro filósofo le otorga a estas no-personas cierto valor, con la introducción del término persona social. ${ }^{27}$ Engelhardt desea reconocer al ser humano no-persona 'como si' fuera una persona, cuando no lo es en

${ }^{27}$ Ver Engelhardt, "Medicine and the Concept of Person", op. cit., p. 176-7 y The Foundations..., op. cit., p. 146 s. 
sí. Este reconocimiento le otorga, por procuración, derechos relativos a la condición de persona, incluyendo el de la protección de la vida. Sin embargo, Engelhardt plantea ciertas condiciones. Con vistas a acceder al club de persona social, el ser humano no-persona debe ser capaz de comprometerse realmente en una 'interacción social mínima'. ${ }^{28}$ Quien esté desprovisto de esto -como, por ejemplo, el niño con anencefaliano puede ser considerado como una persona social. Aparte del carácter arbitrario $^{29}$ de este criterio de interacción, Engelhardt permanece muy vago en la descripción del concepto de interacción, como también de los adjetivos 'social' y 'mínimo'. No menciona tampoco criterios racionales que fundarían la línea de demarcación de lo 'social mínimo'.

Tras haber elaborado el concepto de persona moral siguiendo el modelo kantiano, Engelhardt construye la noción de persona social siguiendo el modelo utilitarista y consecuencialista. Esta noción es, en efecto, esencialmente una 'construcción utilitarista' ${ }^{30}$ que no tiene como meta principal el bien del ser humano no-persona, sino el de la persona moral.

El sentido social de persona expresa una manera de tratar ciertos casos de la vida humana con vistas a garantizar la vida de las personas en sentido estricto. [...] Una persona en ese sentido no es una persona propiamente dicha y no es, así, un objeto de respeto sin reservas. Más precisamente, ciertos casos de la vida humana son tratados como personas por el bien de los individuos que son personas en el sentido estricto. ${ }^{31}$

El reconocimiento de ese ser humano como persona social estaría justificado por producir en las personas morales importantes virtudes

${ }^{28}$ Ver Engelhardt, "Medicine and the Concept of Person", op. cit., p. 176.

${ }^{29}$ Para la ciencia de la haptonomía, el feto, por ejemplo, puede encontrarse sin problemas activamente en una interacción social con su entorno, más particularmente con sus padres. Ver F. Veldman, Haptonomie. Science de l'Affectivité [Haptonomía. Ciencia de la afectividad], 2001, París, PUF, (1989).

${ }^{30}$ Engelhardt, "Medicine and the Concept of Person", op. cit., p. 177.

31 Ibid. 
personales, como la simpatía y la protección ('care’). ${ }^{32}$ Engelhardt menciona también la razón de una prudencia concerniente a la incertidumbre que planea sobre el tema del momento exacto en el que el ser humano reconocido como persona social se convertiría en una persona moral.

No me parece que la introducción del término persona social excluya categóricamente, como pretende Engelhardt, la eutanasia y la utilización con fines experimentales de ciertos niños normales, de niños o de adultos mental o físicamente deteriorados, como los seniles. En efecto, como la atribución de la condición de persona social a $\mathrm{X}$ depende principalmente de un cálculo utilitarista y consecuencialista con vistas a la maximización del bien de los sujetos morales, podría ocurrir lógicamente que personas morales genitoras conciban que la muerte de su hijo o de su padre no-persona, de quienes ellos son propietarios, conduciría a una maximización de la felicidad. Recordemos que, según la ética secular, cada persona moral, respectivamente cada comunidad particular tiene una legitimidad ética para actuar de acuerdo con su propia concepción ética. Por lo tanto, desde el punto de vista secular, no puede haber prohibición absoluta y universal de la utilización con fines experimentales de un ser humano no-persona, o de la eutanasia, por parte de personas morales o de comunidades individuales. Además, la atribución de la condición de persona social es a partir de esto extremadamente relativa dentro del contexto de una ética secular. El ser humano no-persona sigue siendo principalmente un objeto que puede ser utilizado como un simple medio por sus propietarios, incluso por una sociedad anónima. Está a merced del cálculo utilitarista y consecuencialista, con vistas al bien de las personas morales que son las únicas que tienen derecho a la vida. Los derechos relativos a la persona social no son creados por la comunidad de todos los extranjeros morales que son sujetos de la ética secular, sino, como lo reconoce Engelhardt, por comunidades particulares. ${ }^{33}$

${ }^{32}$ Ver Engelhardt, The Foundations..., op. cit., p. 147.

33 "Persons who are moral agents have rights that are integral to the very character of general secular morality. The rights of persons in a social sense are created by particular communities", ibid, p. 150. 
El hecho de mantener con vida a niños gravemente impedidos física o mentalmente -como, por ejemplo, con anencefalia-incluso si esto se llevara a cabo con poco gasto, en opinión de Engelhardt incurre en una incomprensión moral. Sin embargo, nuestro autor no sugiere la eutanasia activa, pues esto tendría, según él, costos sociales muy elevados, sino la aplicación de una ley similar a la de Solón, a saber, la interrupción del tratamiento:

Se puede decidir salvar, cayendo en grandes gastos, a un niño que será probablemente normal, pero no a alguien cuyos impedimentos físi$\cos \mathrm{y}$ mentales futuros constituirían incluso más peso psicológico y económico, sumados a los costos desmesurados implicados en el hecho de salvar la vida del niño. ${ }^{34}$

Es necesario precisar varios puntos. La afirmación 'se puede decidir' es proferida con un trasfondo de utilitarismo y de consecuencialismo por parte de los padres propietarios del niño llamado 'normal' o 'impedido'. Sin embargo, se podría sostener la legitimidad moral por parte de estos mismos padres de salvar a su hijo -incluso si está profundamente impedido-si se consideran preparados para asumir los costos y el peso psicológico de esto. Engelhardt tendría que estar necesariamente de acuerdo, incluso si plantea que se trataría de un acto moralmente incomprensible. $^{35}$ ¿Sobre qué criterios se basa para emitir semejante juicio? ¿Acaso no está emitiendo un juicio universal que no podría ser válido dentro de una ética secular? Además, la afirmación de acuerdo con la cual la eutanasia tendría costos sociales más altos es más bien, en mi opinión, una hipótesis desprovista de fundamento argumental en el contexto particular del párrafo citado. Si Engelhardt quiere ser fiel a estas premisas, debe lógicamente sostener la legitimidad ética de que ciertas perso-

${ }^{34}$ Engelhardt, "Infanticide in a Post-Christian Age", p. 85. "Anencephalic children. When no or little brain is present and there is no possibility of sentient life, not to mention personal life, it would be a moral misunderstanding to try to sustain such an infant, even if it could be done cheaply and effectively. There is no person in the body to be benefited", op. cit., p. 83.

${ }^{35}$ Ver ibid., p. 83. 
nas -los padres o una sociedad anónima- puedan libremente decidir aplicar la eutanasia a su niño gravemente impedido o a su padre senil, e incluso utilizarlos como un simple medio con vistas a realizar experimentos. Nuestro autor afirma al respecto que

la condición secular del infanticidio está implícita. Es la resultante de la incapacidad de la moral secular de justificar una explicación general autorizada plena de contenido para la condición moral de los fetos o de los niños pequeños. Este fracaso limita la autoridad moral secular del Estado para intervenir. Una proscripción exigiría una jerarquización particular autorizada de los daños y de los beneficios. Esto sólo podría justificarse en el interior de una visión moral particular. La conclusión es muy desagradable. El equivalente de la no intervención ateniense y romana en lo concerniente al infanticidio es inevitable, dada la autoridad moral secular limitada del Estado. La prohibición legal del infanticidio sobre la base de la consideración de la beneficencia (por ejemplo, para realizar una concepción particular del bello arte de ser padre) exigiría el establecimiento de una autoridad gubernamental para rebasar la autonomía parental cuando no se le hubiera hecho ningún daño a una persona en el sentido estricto del término. La dificultad consiste en mostrar cómo proteger la moralidad de los demás individuos o comunidades en el marco de la imposición de la autoridad moral secular desde un punto de vista particular. Quienes defienden el infanticidio verán la ventaja de su práctica como más importante que los bienes que se acumulan a causa de su prohibición. En razón de la centralidad del principio de permisividad, la prueba de cargo está del lado de los interventores en cuanto a mostrar que las acciones parentales podrían estar prohibidas, y no concierne a los padres demostrar que tienen la libertad de actuar. ${ }^{36}$

\section{Hacia una antropología unitaria}

Ciertamente que Engelhardt tiene razón al sostener que una comunidad particular no podría imponer por la fuerza y la inquisición su propia

${ }^{36}$ Engelhardt, The Foundations..., op. cit., p. 271. 
ética particular a expensas de las demás comunidades. A pesar de haber renunciado a fundar racionalmente una ética universal, nuestro autor ubica en el corazón de su pensamiento un concepto universal: la persona moral, y la distinción ontológica entre ésta y los seres humanos. Esta separación expresa una concepción dualista del individuo humano, a saber, entre la dimensión biológica, el cuerpo humano, por un lado $\mathrm{y}$, por la otra, las facultades llamadas superiores que provienen del espíritu. La pertenencia biológica y genética a la especie humana mantiene una relación extrínseca con la auto-conciencia y la racionalidad. El cuerpo humano no esta entendido como una parte integral de la persona que la revelaría en un tiempo y un espacio particulares.

Engelhardt comete, además, un error epistemológico, en la medida en que le da una condición ontológica a unos entia rationis. Sostiene que la propiedad es el referente, y se opone así a la tesis que sostiene que la persona denota el referente y que la auto-conciencia, por ejemplo, denota una propiedad particular de un referente, a saber de la persona. Dicha propiedad manifiesta la naturaleza de la persona. El ser humano se despliega como persona que se puede manifestar, pero no necesariamente, en su actuar por el ejercicio de propiedades. Su posesión en acto en el sentido particular de su ejercicio no es un requisito para ser en acto una persona. Los seres humanos desprovistos del ejercicio de las propiedades llamadas personales son plenamente personas, sólo que deficientes. La aptitud para ejercer estas propiedades, a causa de la naturaleza propia, y en un momento dado de su historia, es una condición necesaria para calificar al ser humano como persona. $\mathrm{Su}$ eventual ejercicio concreto es secundario y no tiene incidencia en el otorgamiento de la condición de persona.

Podemos distinguir entre lo que el 'siendo' es (ontología del ser) y la actualización de lo que él es en plenitud (ontología del no ser todavía). Esta última sólo se despliega a partir de un ser en acto. La diferencia entre un adulto llamado 'normal' y un recién nacido, un embrión o un cigoto no reside en su ser - persona, sino en el grado de despliegue de sus propiedades llamadas personales. Los conceptos de potencialidad y de sustancia están íntimamente vinculados. Otorgado a un vivo, 
el primero presupone ante todo un fundamento metafísico: la sustancia que define a un ser vivo particular.

Podemos diferenciar, por una parte, una forma sustancial presente desde el comienzo de la existencia humana, es decir, un 'siendo' mínimo-realizado, un no ser todavía en plenitud, y por el otro lado, el actuar del ser humano, que lo orienta de manera determinada-indeterminada, es decir, libre, hacia un fin último inscrito en el fondo de su naturaleza y que implica la libre actualización de la totalidad de su poder-ser. El ser humano se encamina hacia su realización -tanto por naturaleza como por el actuar libre y por don-en el plano del ejercicio de las propiedades inherentes a su naturaleza, hacia un ser-pleno, hacia un poseer-plenamente. El ser humano tiende a desplegar lo que es, a realizar su naturaleza, en todas sus dimensiones. A partir de un estado determinado, identificado con su concepción, la persona está orientada hacia la excelencia de su naturaleza a través de su cuerpo, su inteligencia y su voluntad. Esta meta hacia la excelencia de la naturaleza humana, a su acabamiento, presupone un ser-sujeto que haga posible este impulso, este despliegue.

\section{Conclusión}

Sobre el trasfondo de la distinción entre el acto y el ejercicio, o, para retomar un término más técnico, entre el acto primero y el acto segundo, podemos sostener que un individuo que no ejerza, por diversas razones, propiedades llamadas personales, está sin embargo en condiciones de ser en acto -y más particularmente en acto primero- de existencia personal. La potencialidad de ejercer dichas propiedades está presente desde la concepción del individuo, y las propiedades podrán ser ejercidas concretamente en lo cotidiano si ninguna influencia exterior y ningún mal funcionamiento interior al sujeto viniera a trabar el desarrollo de la persona. Tal comprensión de la persona hace posible concebir no solamente al cigoto, al embrión y al recién nacido como personas, sino también al senil, al comatoso y al individuo seriamente impedido 
mental y/o físicamente. Su naturaleza humana es plenamente personal, sólo que es deficiente en cuanto en el ejercicio de ciertas propiedades llamadas personales, que debería poder ejercer si las circunstancias normales estuvieran dadas en un instante particular de su desarrollo.

Una de las consecuencias de esta posición reside en el hecho de que la persona lo es en acto, al mismo tiempo que tiene a su disposición -en todo momento de su existencia- la potencialidad de desarrollarse finalmente en cuanto persona. Entonces, es sujeto de derechos fundamentales en virtud de su mismo ser de persona, es decir, que el individuo humano no necesita ejercer un comportamiento que exprese las propiedades llamadas personales para tener un pleno derecho a la vida.

No debemos tomarnos a la ligera el desafío de una definición secular de la persona para la ética lanzado por Engelhardt, refugiándonos detrás de la afirmación de que estamos ante una moda que pasará. Yo no creo que se trate de una moda, sino de un proceso de cambio fundamental de nuestra comprensión ética occidental, que hasta ahora ha tenido su fundamento en la tradición judeo-cristiana. La distinción entre ser humano y persona subyacente al llamado apremiante de instaurar una ética secular es defendida por un número creciente de filósofos, principalmente anglosajones y alemanes. La introducción de una ley de Solón para el caso de seres humanos profundamente impedidos mental y/o físicamente, y también de los seniles, se abre paso lentamente en la conciencia pública, a pesar de que dicha ley sigue siendo hoy un tabú. La solución de este desafío de cultura y de sociedad no podría reposar sobre una actitud fideísta como la de Engelhardt, quien es ortodoxo creyente, actitud que otorga una primacía absoluta y totalizadora a la experiencia espiritual y a la sola fe, sino que debe buscarse en una urgente reflexión filosófica antropológica. 
La reproducción total o parcial de este artículo se podrá hacer si el ITAM otorga la autorización previamente por escrito. 